\title{
Stone fragmentation by ultrasound
}

\author{
S K SHRIVASTAVA* and KAILASH ${ }^{\dagger}$ \\ Department of Physics, Institute of Basic Sciences, Bundelkhand University, Jhansi 284 128, India \\ ${ }^{\dagger}$ Physics Department, BNV College, Rath (Hamirpur) 210 431, India
}

MS received 29 March 2004; revised 20 May 2004

\begin{abstract}
The presence of kidney stone in the kidney causes discomfort to patients. Hence, removal of such stones is important which is commonly done these days, non-destructively, with lithotripters without surgery. Commercially, lithotripters like extra-corporeal shock wave lithotripters (ESWL) made by Siemens etc are in routine use. These methods are very cumbersome and expensive. Treatment of the patients also takes comparatively more time because of more number of sittings. Some delicate nerves and fibres in the surrounding areas of the stones present in the kidney are also damaged by high ultrasonic intensity used in such systems. In the present work, enhancement of the kidney stone fragmentation by using ultrasound is studied. The cavitation bubbles are found to implode faster, with more disintegration efficiency of the lithotripters, which give better treatment to the patients.
\end{abstract}

Keywords. Stone fragmentation; ultrasound; ESWL.

\section{Introduction}

Disintegration of renal calculi, non-invasively, with the use of lithotripters is increasing day by day (Singh et al 1999). Renal calculi are heterogeneous structures containing mainly oxalates, phosphates, uric acid, gypsum and alpha quartz. Commercial extra corporeal shock wave lithotripters (ESWL) are used in the hospital for the treatment of kidney stones as well as gall bladder stones (Singh et al 1999). High intensity focused shock waves are applied to the stone surface for stone fragmentation into small sand like particles, which are flushed out with urine. Efforts have been made for improving the efficiency of these systems to increase the rate of fragmentation of the stones. The aim of the work is to study theoretically cavitation bubbles to implode faster and to release more energy on the stone surface for its early and quicker fragmentation. Today the treatment of kidney and ureteral stones with extracorporeal shock waves is the treatment of the first choice. Modern lithotripters work without a bathtub and without anesthesia (figure 1).

\section{Theoretical approach}

The instantaneous intensity, $I_{\mathrm{f}}$, at the focus, if the pressure and the particle velocity are considered to be in phase at the focus of the ESWL, is given by

$$
I_{\mathrm{f}}=\frac{p^{2}}{\rho c}
$$

\footnotetext{
*Author for correspondence

**The ratio of $I_{\text {spta }}$ and $I_{\text {sppa }}$ is duty factor; duty factor is defined as the ratio of pulse duration, $t_{\mathrm{d}}$, and pulse repetition period (s).
}

Here ' $p$ ' is the instantaneous acoustic pressure, $\rho$ ' the density of the medium and ' $c$ ' the speed of sound in water $(\sim 1100 \mathrm{~m} / \mathrm{s})$.

The product of the pulse intensity integral (PII) as per the American Institute of Ultrasound in Medical (AIUM)/ National Electrical Manufacturers Association (NEMA) definition and the pulse repetition frequency (RPF) set to $1 \cdot 0 \mathrm{~Hz}$, is the spatial-peak temporal-average intensity $\left(I_{\text {spta }}\right)$. The spatial-peak pulse-average intensity $\left(I_{\text {sppa }}\right)$ is the ratio of the calculated value of the PII and the pulse duration $\left(t_{\mathrm{d}}\right)$ defined as

$$
t_{\mathrm{d}}=1 \cdot 25\left(t_{2}-t_{1}\right)
$$

$t_{1}$ and $t_{2}$ are the times at which PII exceeds $10 \%$ to $90 \%$ of its total value. The ratio of $I_{\mathrm{spta}}$ and $I_{\mathrm{sppa}}$ is $t_{\mathrm{d}}$ and it is reported as $0 \cdot 3$ to $10 \mu$ s (Coleman and Saunders 1989)**. For a typical $1 \mathrm{~cm}$ diameter of the kidney stone, a wide range of focal beam areas permit focusing on a small part of the stone or completely enclosing the stone within the focal area.

The radiation pressure exerted on the stone placed in the path of the sound wave is

$$
P=I \times c P
$$

where $P$ is acoustic pressure, $I$ the acoustic intensity and $c$ the sound velocity. The ultrasonic intensity, $I_{\mathrm{f}}$, according to the theory of focusing, at the focal lobe is given by (Chaussy 1982)

$$
I_{\mathrm{f}}=\frac{0 \cdot 84 W}{\pi / 4 \times d_{\mathrm{f}}^{2}},
$$

where ' $W$ ' is the total ultrasonic radiation power at the focal lobe and $d_{\mathrm{f}}$ the focal diameter given as

$$
d_{\mathrm{f}}=1 \cdot 22 \lambda \text {. }
$$


When the acoustic power is measured by power management system like radiation pressure balance, the power is given by

$$
W=\frac{F \times c \times 10^{-7}}{2 \cos ^{2} \alpha},
$$

where ' $F$ ' is the radiation force in dynes, ' $\alpha$ ' the angle between the normal to the target surface and ultrasonic beam axis, which is generally taken as $30^{\circ}$ for a float target otherwise as $0^{\circ}$ or $180^{\circ}$ for flat target surface and ' $c$ ' the ultrasonic velocity of water. Pressure is defined as thrust per unit area,

$$
P=\frac{F}{a_{\mathrm{f}}},
$$

where ' $a_{\mathrm{f}}$ ' is the area of focal lobe in $\mathrm{cm}$ and ' $F$ ' the force or thrust in dynes. When $a_{\mathrm{f}}$ is taken in $\mathrm{m}^{2}$ and $F$ in Newton, then pressure ' $P$ ' is defined in Pascal.

According to Rayleigh theory, the radius of single cavity is correlated by

$$
t_{\mathrm{c}}=\frac{0 \cdot 915 R \sqrt{\rho}}{\rho_{0}},
$$

where ' $t_{\mathrm{c}}$ ' is the bubble decay time needed by a single bubble with an initial maximum radius, $R$, to collapse in a medium with a density, $\rho$, under a hydrostatic pressure, $\rho_{0}$.
The potential energy ' $E$ ' in a single cavitation bubble is proportional to its volume and is theoretically given by

$$
E \propto R^{3} .
$$

Hence

$$
E \propto t_{\mathrm{c}}^{3}
$$

Therefore, maximum bubble radius is proportional to the cavitation lifespan

$$
R \propto t
$$

Thus the bubble size has direct relationship with its life and if the size of bubble is large, its life is more and vice versa.

\section{Results and discussion}

The ultrasonic output data of commercial ESWLs like Siemans lithostar is shown in table 1 . These data were given by the manufacturers and also by Coleman and Saunders (1989). Focal transducers with different acoustic lenses were made by using metals and hard plastics (Apfel 1981; Singh et al 1988). The acoustic intensity with different power levels was determined at different focal lengths with different types of developed lenses. The pressure generated by the ultrasonic wave was evaluated by (7). The acoustic intensity was found to increase the pressure amplitudes of the focused ultrasonic wave at the experimental

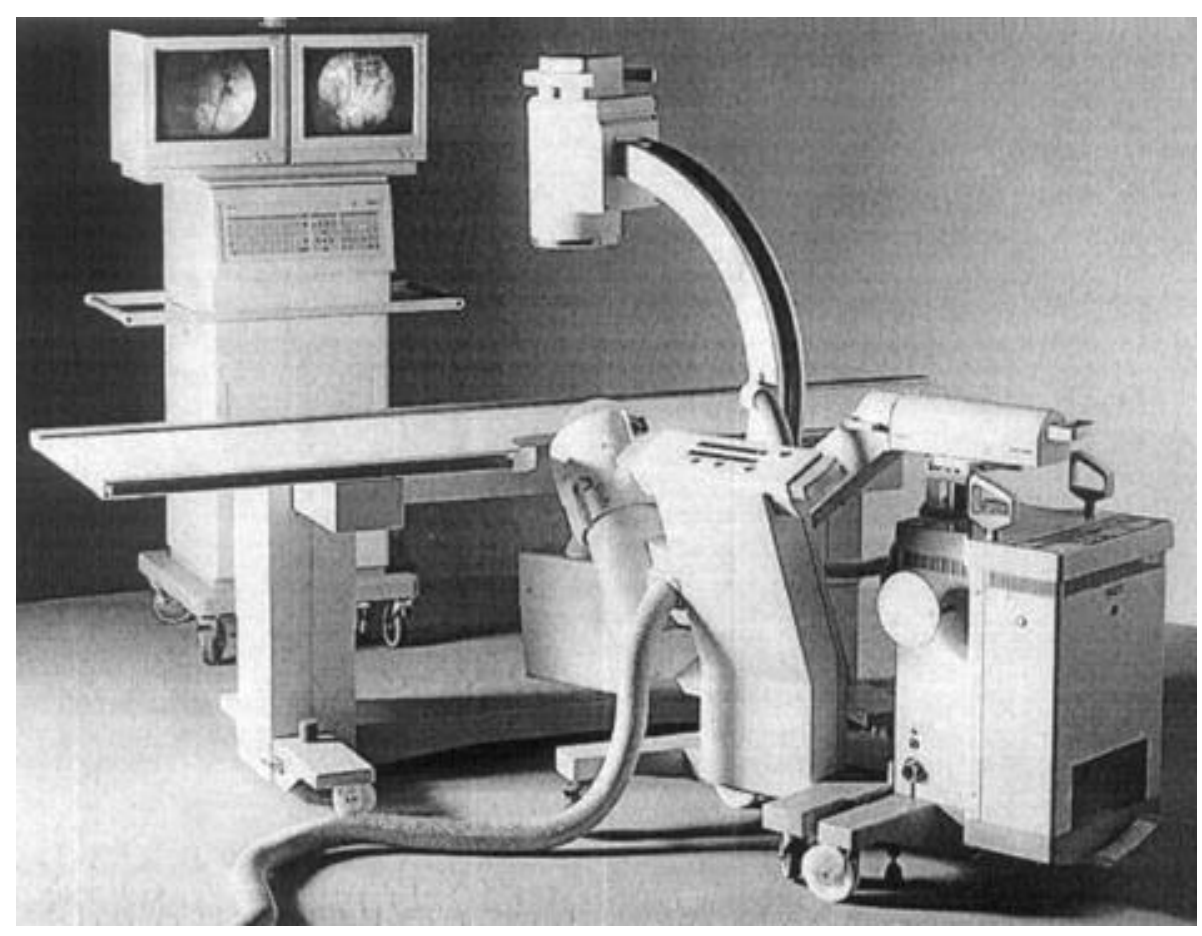

Figure 1. Modern lithotripters (Litho Tron, HMT) for the treatment of kidney and ureteral stones with extracorporeal shock waves. 
Table 1. Ultrasonic out put data of commercial (ESWLs).

\begin{tabular}{lll}
\hline 1 & Negative STPT $\left(\mathrm{p}^{-}\right)$ & $2 \cdot 8 \mathrm{MPa}$ to $9.9 \mathrm{MPa}$ \\
2 & Positive STPT $\left(\mathrm{p}^{+}\right)$ & $20 \mathrm{MPa}$ to $114 \mathrm{MPa}$ \\
3 & Pulse average intensity $\left(I_{\text {sppa }}\right)$ & $6.6 \times 10^{7} \mathrm{Wm}^{-2}$ to $1.24 \times 10^{9} \mathrm{Wm}^{-2}$ \\
4 & Temporal-average intensity $\left(I_{\text {spta }}\right)$ & $5 \times 10^{2} \mathrm{Wm}^{-2}$ to $1 \times 10^{4} \mathrm{Wm}^{-2}$ \\
5 & Focal beam areas & $0.03 \times 10^{-4} \mathrm{~m}^{2}$ to $3.8 \times 10^{-4} \mathrm{~m}^{2}$ \\
6 & Max. energy & $0.10 \mathrm{~J}$ \\
7 & Total energy & 0.002 to $0.03 \mathrm{~J}$ \\
\hline
\end{tabular}

stone surface. The additional pressure induced by the ultrasonic wave generates more pressure on the cavitation bubbles on the stone surface due to the normal shock wave pressure from the ESWL. High intensity ultrasound concerning liquids involves the use of cavitation. When a liquid subjected to rapidly alternating pressures of high amplitudes, which can be brought about by the use of sound of sufficient intensity, cavitation takes place in the liquids. When sound travels through a small region in a liquid, the liquid in the region is compressed during its positive half cycle and during its negative half cycle, it expands. The molecules of the liquid are wrenched apart and voids are created when the acoustic pressure is greater than hydrostatic pressure. The voids contain only vapour of the liquid. These voids expand during the negative part of the pressure cycle and then collapse abruptly. Due to this collapse, energy is released at an enormous rate which generates the shock waves. This additional pressure induced through the focused ultrasound, increase further the acoustic pressure at cavitation bubbles inside the pores of the stone. The resultant pressure is greater than the hydrostatic pressure, giving rise to quicker collapse of the bubbles and hence quicker disintegration of stones.

\section{Conclusions}

The physics behind enhancement of the disintegration of the kidney stones by using lithotripters, ESWL, has been studied. The study would help in the development of better lithotripters with better efficiency.

\section{References}

Apfel R E 1981 Methods of experimental physics (ed.) P E Edmonds (New York: Academic Press) 19 pp 355-411

Chaussy C (ed.) 1982 Extracorpeal shock wave lithotripters (Basel: Karger)

Coleman A J and Saunders E 1989 UIt. Med. Biolog. 15213

Singh V R, Bindal V N and Singh G 1980 Ultrasonics 1828

Singh V R, Agrawal R and Sud S P 1988 J. Instn. Engrs. (India) 695

Singh V R, Lafaut J P, Wevers M, Vincent C and Baert L 1999 lCEU-99, New Delhi, pp 418-421 